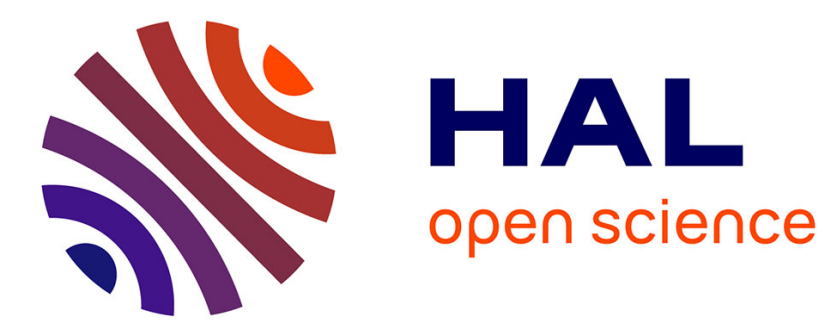

\title{
The atomic structure of the decagonal phase and its approximants as random coincidence networks
}

D. Romeu, J.-L. Aragon, M. Torres, J.-L. Verger-Gaugry

\section{To cite this version:}

D. Romeu, J.-L. Aragon, M. Torres, J.-L. Verger-Gaugry. The atomic structure of the decagonal phase and its approximants as random coincidence networks. Journal of Physics: Condensed Matter, 1993, 5, pp.7785-7796. hal-03134289

\author{
HAL Id: hal-03134289 \\ https://hal.science/hal-03134289
}

Submitted on 11 Feb 2021

HAL is a multi-disciplinary open access archive for the deposit and dissemination of scientific research documents, whether they are published or not. The documents may come from teaching and research institutions in France or abroad, or from public or private research centers.
L'archive ouverte pluridisciplinaire HAL, est destinée au dépôt et à la diffusion de documents scientifiques de niveau recherche, publiés ou non, émanant des établissements d'enseignement et de recherche français ou étrangers, des laboratoires publics ou privés. 


\title{
The atomic structure of the decagonal phase and its approximants as random coincidence networks
}

\author{
D. Romeu ${ }^{\ddagger}$, J.-L. Aragon ${ }^{\ddagger}$, M. Torres* ${ }^{*}$, J.-L. Verger-Gaugry ${ }^{\dagger}$, \\ ¥ Instituto de Fisica, UNAM. Apartado Postal 20-364.01000 Mexico, DF. Mexico \\ * Instituto de Electrónica de Comunicaciones. CSIC. Calk Serrano 144. 28006 Madrid, \\ Spain \\ ${ }^{\dagger}$ LTPCM/ENSEEG/INPG, CNRS UA 29, BP 75, Domaine Universitaire, 38402 Saint \\ Martin d'Hères, France
}

\begin{abstract}
It is shown that the decahedral recursive model (DRM) explains at atomic level the structure of the decagonal (0) phase and associated approximant structures as random coincidence networks (RCNS), random packings of clusters satisfying DRM energy minimizing rules. RCNS are degenerate random tilings that become quasiperiodic when entropic disorder is maximized at high tempmure through phason flips requiring small atomic movements. Such phason flips can change the relative ratio and geometry of tiles, making transformations between systems using different tile sets possible. Electron microscopic observations for AlCuCoSi. AlCuFeCr, AlCoCu and AlPdMn systems are reproduced, including the observed Fibonacci sequenced lines in the former.
\end{abstract}




\section{Introduction}

At present, there remain two basic models attempting to describe the basic structure of quasicrystals: the perfect quasicrystal model (PQM) [1-3], and the random tiling model (RTM) [4]. The former is based on the Penrose tiling and assumes that quasicrystals are deterministic packings of unit celts while the latter describes them with only average quasiperiodicity. PQM assumes that microscopic forces, manifesting themselves as matching rules between tiles, force a quasipertodic ground state. In practice, this requires frequent back-tracking to undo wrong choices making further growth impossible without violating these rules, so, at least for rapidly cooled quasicrystals, PQM does not appear plausible. The RTM, on the other hand, regards quasicrystals as a random packing of tiles that follows a relaxed set of matching rules. In this scheme, the same energy is assumed for all possible tilings and the equilibrium state, not necessarily a ground state, is assumed to be one of maximum entropy at high temperature,

Experimentally, it is difficult to decide which model is correct since there is apparently conflicting evidence supporting both views. On the one hand, it has been reported that I$\mathrm{Al}_{63.5} \mathrm{Cu}_{12.5} \mathrm{Fe}_{24}$ [5], D-AI $\mathrm{A}_{63} \mathrm{Co}_{17.5} \mathrm{Cu}_{17.5}$ [6], D- $\mathrm{Al}_{65} \mathrm{CO}_{20} \mathrm{Cu}_{15}$ [7] and D- $\mathrm{Al}_{65} \mathrm{Cu}_{20} \mathrm{Co}_{15} \mathrm{Si}_{2}$ [8] undergo crystal-quasicrystal phase transformations at high temperature, the former being reversible. Conversely, other experiments on the $\mathrm{I}-\mathrm{Al}_{65} \mathrm{Cu}_{20} \mathrm{Fe}_{15}$ system report a stable quasiperiodic phase at all temperatures [9].

In this paper we will show, using the resuits of the decahedral recursive model (DRM) [10-14], applied to decagonal D phases $[15,16]$, that the D phase, and all observed approximants, are completely describable by a random accretion of clusters following a simple set of packing rules. This description is basically consistent with a random tiling 
scenario [4] where a measure of the energy has been introduced by DRM through packing rules. It will be shown that a quasiperiodic state is obtained when disorder is maximized (at high temperature) through random phason flips produced by small atomic displacements between adjacent $\mathrm{O}$-point sites (see below). Also, the atomic structure of several D systems and their associated approximants, namely $\mathrm{AlCuCoSi}, \mathrm{AlCuFeCr}[17], \mathrm{AlCoCu}[7]$ and AlPdMn [18], will be explained, including the presence of Fibonacci sequenced lines in the former.

Other D atomistic models have been proposed [19-23]. Generically, the difference from the DRM lies in the fact that their structures have been formulated to describe specific alloys, in contrast with DRM structures derived from general principles. As a consequence other models do not offer an explanation for the origin of the basic clusters, the different periods and the general occurence of D wheels of bright spots in all D systems. Also, the formation mechanism and structure of associated crystalline approximants is left unexplained. In particular, Burkov's model was specifically designed for D-AlCuCo, based on the observation of HREM micrographs. Although Burkov's basic cluster allows two different degrees of interpenetration, it can produce the same long range configurations of the DRM structures, which allows only one using a smaller basic cluster. The principal difference between Burkov and DR models lies in their basic cluster, the former having only two atomic layers whilst the latter has some of its atoms at slightly elevated positions defining layers A and B (see below). Note that atoms at elevated positions have been observed by scanning tunnelling microscopy [24].

\section{The DRM model}

DRM is a general atomistic model that simulates crystalline and quasicrystalline atomic structures of any symmetry [13-16]. Following a small set of energy minimizing rules, it describes how atoms aggregate to form low-energy clusters (decahedral stage) and how these coalesce (recursive stage) to form macroscopic structures by minimizing the clustercluster interfacial energy. The recursive stage is based on the coincidence sites lattice model [25] and it states that there are sites in the structure (called O-points) with the property of being preferred nucleation centres for atomic clusters. In the I and D cases, O-points are centres of (normally distorted) icosahedra $[13,15]$.

Nucleation of clusters around $\mathrm{O}$-points produces a high number of atomic coincidences between clusters and, thus, low-elastic-energy structures. Some O-points are special in that they give rise to a larger percentage of coincidences than others, and have the same surroundings up to a larger volume. The atoms in this volume define a repetitive pattern called a basic cluster that composes the structure, so special O-points (network nodes) are simply called cluster centres. DRM structures are therefore semi-random (randomness is limited by coincidence requirements) networks of cluster centres (or nodes, defining the lattice or quasilattice), decorated with interpenetrating basic clusters. Since every atom belongs to a basic cluster, the maximum density condition is satisfied and a tiling is defined [4] with edges linking network nodes.

\section{The D phase}

Figure 1(a) shows, plane by plane, the basic cluster that yields the DRM D structure of $0.4 \mathrm{~nm}$ period, up to the nearest $\mathrm{O}$-points in each plane. The cluster, obtained from first principles 

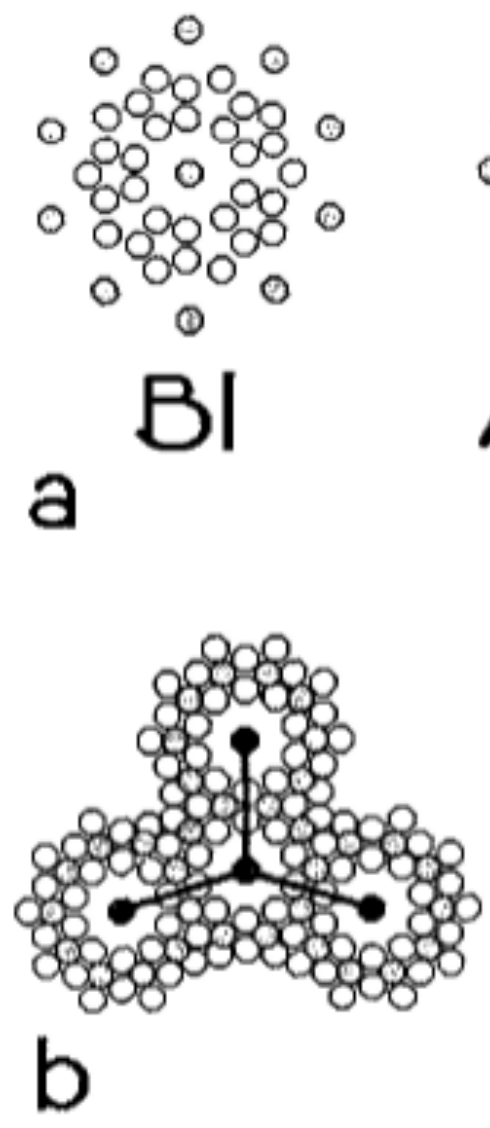
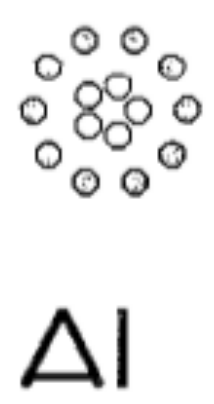
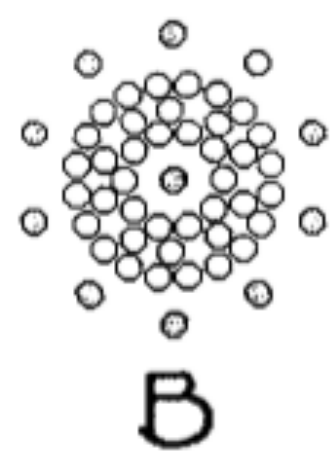

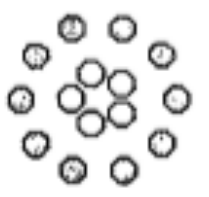

$\triangle$

Figure 1. (a) Each of the four planes BL, AI, B, A of the $0.4 \mathrm{~nm}$ basic cluster up to closest O-points (shaded), (b) Left: maximum-coincidence (100\%) configuration of basic clusters. Note that every atom belongs to at least one basic cluster. The shaded circles are O-points of planes $\mathrm{Al}$ and $\mathrm{A}$, above and below plane BI. Centre and right: Planes $\mathrm{BI}$ and $\mathrm{Al}$ of a defected RCN. Note non-coincident overlap of atoms inside 36 and $72^{\circ}$ mombi.

by Romeu $[10,15]$, has atoms at the sites proposed by Kortan et al [24] and Becker and Kortan [26], based on their scanning tunnelling observations. Other clusters yielding 0.8, 1.2 and $1.6 \mathrm{~nm}$ periods were also found. All clusters are subdivided in fundamental units of length $0.4 \mathrm{~nm}$ units along $c$, following their own packing rules, also leading to randomness in the periodic direction $[15,16]$ and giving rise to all observed periodicities in D systems. The atoms in the $0.4 \mathrm{~nm}$ cluster are distributed over four planes labelled A, AI, B and BI (a fundamental unit) which, when stacked in the sequence ...,-BI-AI-B-A-BI- ... along the 10 -fold $c$ axis, define a $0.4 \mathrm{~nm}$ period $\mathrm{D}$ column. Note that this configuration does not have a mirror plane as is commonly accepted. Such a mirror is also possible under DRM rules [15] yielding a stacking sequence ...-BI-AI-B-AI-BI-.... This, however, introduces faults in the stacking sequence of distorted icosahedra along the $c$ axis, increasing the elastic energy of the system [15]. In view of the above, and the fact that diffraction patterns of a large enough cluster [27] from a periodic stacking of planes ...-BI-AI-B-A-BI-,... have also a $10 / \mathrm{mmm}$ symmetry with extinctions in accordance with experimental observations [28], we believe the unmirrored sequence to be more adequate. The reader shoud note, however, that this is not a crucial point in what follows since the atomic sites that make planes $\mathrm{A}$ and AI different do not affect the coincidence relationships between clusters, making the results discussed in this paper independent of the existence of the mirror plane. The distance between successive planes is $b=5^{-1 / 2}$ in reduced units, approximately equal to 

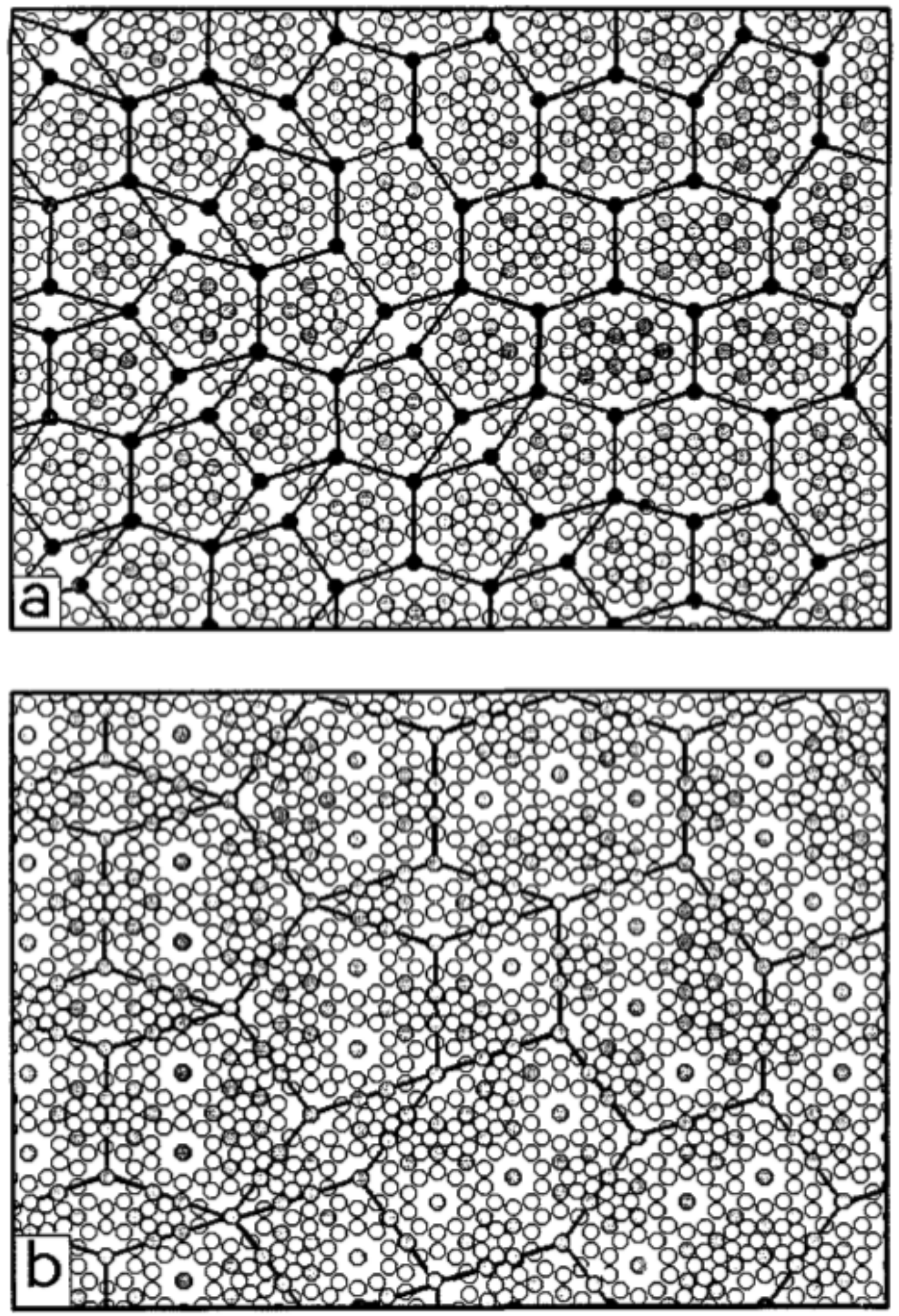

Figure 2. (a) $O_{1}$ (right) and $O_{2}$ (left) approximants of AlCuFeCr. The tile edge length is $1.2 \mathrm{~nm}$. (b) $O_{1}^{\prime}$ (right) and $O_{2}^{\prime}$ (left) approximants of AlCoCuSi with $2 \mathrm{~nm}$ edge length. (c) Approximant of AlPdMn with different cell decorations. On the left isolated cells are shown, Cluster centres may occupy either of the two positions inside the cell at the top or, equivalently. along the edges of the bottom cell. (d) Monoclinic approximant of AlCuCo. Cluster centres are filled and $\mathrm{O}$-point sites shaded. 

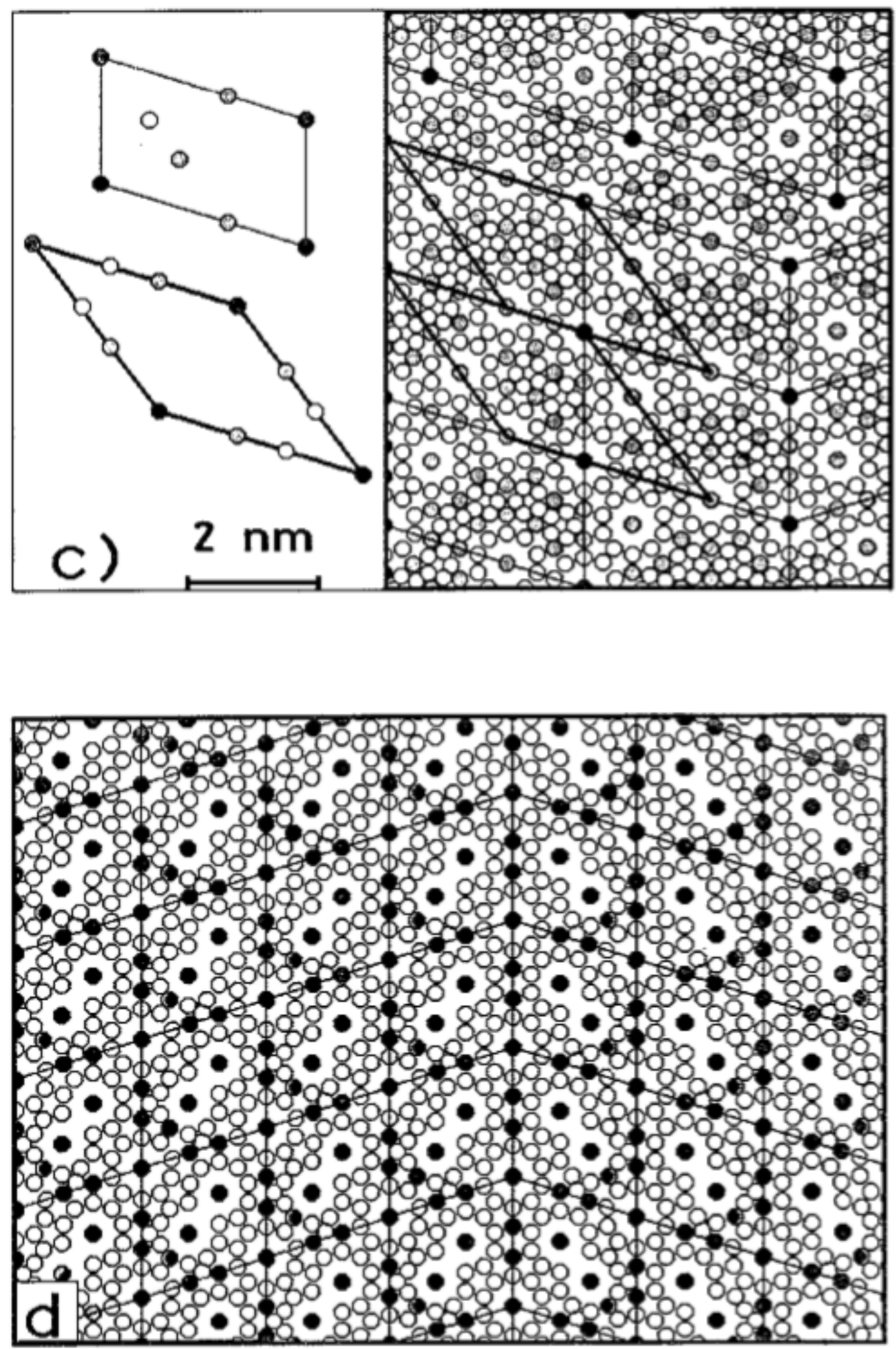

Figure 2. (Continued) 
$0.109 \mathrm{~nm}$. Cluster planes $\mathrm{A}$ and $\mathrm{AI}$ have $\mathrm{O}$-points defining a decagon (DA) of circumradius $6 b$ around the $c$ axis, while those of planes B and BI define a decagon (DB) of circumradius $12 b \cos 18^{\circ}$, equivalent to a cluster radius of about $1.24 \mathrm{~nm}$. Note that due to periodicity, $\mathrm{O}$-points are located along $\mathrm{O}$-lines parallel to $c$, and that the difference between planes $\mathrm{A}(\mathrm{B})$ and $\mathrm{AI}(\mathrm{BI})$ is that the they have inverted (irregular) pentagons at the same $x, y$ positions.

\subsection{Packing rules}

A macroscopic structure can be grown by laterally shifting the basic cluster into the nearest available O-points so that the number of atomic coincidences is maximized, discarding overlapping atoms [13]. Lateral displacements into any of the $10 \mathrm{DB}$ O-point sites (DB O-lines) give rise to $100 \%$ coincidence between atoms (and O-lines) in all planes, and to a coherent, low-energy, cluster-cluster interface [15]. All DB O-point sites are equally good cluster centres; however, having chosen one, the selection of others is no longer arbitrary, as we shall see below. Small adjustments of the position of some atoms are required ( $\simeq 10^{-2} \mathrm{~nm}$ ) to decrease elastic strain; in practice, final positions are determined by Lennard-Jones relaxation [13]. Interestingly, atoms located at $\mathrm{O}$-point sites require no adjustment. This restriction defines a simple set of packing rules for clusters (not for tiles) that produce decorated networks of cluster centres with maximum atomic coincidence, covering the space completely.

In order to describe the packing rules between clusters, we shall analyse the growth of a maximum-coincidence structure from a single cluster, with the aid of figure I(b). Minimum inter-cluster energy ( $100 \%$ coincidence in all planes) is achieved when linkages between cluster centres (tile edges), make an angle of either $108^{\circ}$ or $144^{\circ}$ as shown in figure l(b) (left), other choices result in energy costly non-coincident atomic overlap and should be considered as structural defects. This is illustrated at the centre and right of figure 1(b), showing a section of planes BI and AI with some defects. Note that the $72^{\circ}$ and $36^{\circ}$ rhombic tiles have non-coincident atoms on planes $\mathrm{AI}$ and $\mathrm{BI}$ respectively, effectively ruling them out of the ground state tiling. The energy cost of the latter is less severe since a relatively low-strain (with lower density) structure can still grow by removing the two overlapping atoms; however, better quasicrystals are obtained from $\mathrm{AlCoCuSi}$ alloys, which do not contain the $36^{\circ}$ thombus (see below), than from AlCoCu alloys, where it is observed. Choosing opposite O-lines $\left(180^{\circ}\right)$ forces the eventual appearance of the above rhombi. In a defect free structure, only three out of the 10 possible DB O-lines, making $108^{\circ}$ and $144^{\circ}$ angles, are allowed. Within this limitation, O-line selection is entirely random since there is no evident physical reason to prefer one coincidence maximizing O-line configuration over another. We shall call the resulting network of cluster centres a random coincidence network (RCN). Later we shall see that O-point sites that are not chosen as cluster centres may eventually become so through phason flips.

\subsection{Approximant structures}

Following the above mechanism, it is possible to build RCNs with small ordered zones or microcrystalline domains. If these domains are large enough, microcrystalline phases with well defined orientation relationships and coherent boundaries may be detectable, as demonstrated in figures 2(a)-(d). For clarity only BI planes are shown, since they permit the tiling to be seen more clearly and the whole 3D structure can be deduced from them (see below). These coincidence networks have been respectively identified as the $O_{1}, O_{2}$, $O_{1}^{\prime}, O_{2}^{\prime}$ [17] of $\mathrm{Al}_{65} \mathrm{Cu}_{20} \mathrm{Fe}_{10} \mathrm{Cr}_{5}$ and $\mathrm{Al}_{65} \mathrm{Cu}_{20} \mathrm{Co}_{15} \mathrm{Si}_{2}$, and the monoclinic $[7,18]$ phases 
of $\mathrm{Al}_{70} \mathrm{Pd}_{13} \mathrm{Mn}_{17}$ and $\mathrm{Al}_{65} \mathrm{Cu}_{15} \mathrm{Cu}_{20}$. Note that the $O_{2}$ phase contains the $36^{\circ}$ rhombus of edge length $1.24 \mathrm{~nm}$. This might explain why $\mathrm{O}_{2}$ occurs in a much smaller proportion than the defect free $O_{1}$ phase [17].

\subsection{Random and quasiperiodic D structures}

A maximally disordered $\mathrm{RCN}$, in the sense of having no discernible micro-domains such as that shown in figure 3(a) (called RCN1), can also be built using the above growth rules. $O$-point sites that could have been chosen as cluster centres are shown shaded. Note that RCN1 shows Fibonacci spaced lines defining a quasiperiodic pentagrid when viewed at a glancing angle. Line segments along vertical Fibonacci lines are drawn at the bottom of the figure as a visual aid. Weak line contrast is observed where closer than normal line segments at the bottom of the figure are found, making it difficult to tell where to place the line. This phenomenon was first observed in $\mathrm{Al}_{65} \mathrm{Cu}_{20} \mathrm{Co}_{15} \mathrm{Si}_{2}$ HREM images [8].

The complete 3D structure will be a periodic $(0.4 \mathrm{~nm})$ stacking (A-B-AI-BI) with $\mathrm{O}$ points at the centres of icosahedra compressed by about $12 \%$ along $c$ to satisfy the periodic requirement. For brevity we will restrict ourselves to the $0.4 \mathrm{~nm}$ period case here, but the same reasoning applies to the $0.8,1.2$ and $1.6 \mathrm{~nm}$ period structures that follow the same coincidence maximizing conditions [15]. Figure 4 shows all planes of a $0.4 \mathrm{~nm}$ period of a D section that we shall call the basic decagon (BD), since the whole quasilattice can be deduced from it. Note the planes AI, above BI, have O-points over BI pentagons and pentagons over BI O-points. Planes B (and A) have O-points at the same $x, y$ positions of planes $\mathrm{BI}$ (and $\mathrm{AI}$ ) and inverted pentagons (rotated by $36^{\circ}$ ) on top of $\mathrm{BI}$ (and $\mathrm{AI}$ ) pentagons, defining columns of distorted icosahedra. These columns run along on O-lines and show up as bright spots in electron micrographs [16], giving rise to the observed wheels of bright spots. Since most D systems show the same D wheel contrast, it appears that they have a similar basic cluster structure, differing only in chemical composition, which in tum determines the nature of the underlying RCN. Under appropriate imaging conditions, RCNs with no discernible microcrystalline domains may show Fibonacci lines because there is a quasiperiodic coincidence network (QCN) of cluster centres associated with them. The QCN associated with RCN1, shown in figure 5, has been drawn over RCN1 with broken lines. Note that the QCN edge lengths are $\tau$ times larger $(\simeq 2 \mathrm{~nm})$, and that the $36^{\circ}$ and $72^{\circ}$ rhombi do not break the $100 \%$ coincidence rule, making the approximant of figure 2 (d) possible. There has been a report of a D phase in AlPdMn [18] where the $36^{\circ}$ rhombus is absent from the $2 \mathrm{~nm}$ tiling; however, closer examination of the micrographs indicates that the tiling may be similar to that of figure 3(b), with some poorly defined D wheels. Note that care must be taken to insure that all QCN tiles are decorated identically, blindly selecting cluster centres from poorly detailed electron micrographs may lead to errors in the determination of the nature of the tiling.

As we have seen, the physical growth rules described above can lead to a particular quasilattice. We have found that this quasilattice can also be generated without appealing to the initial growth rules in three different ways: First by deflating the pentagonal, hexagonal and rhombic tiles composing the QCN as indicated with finer lines in figure 5(a); second by overlapping shifted and rotated copies of the $\mathrm{BD}$ on itself so that the rhombic tiles coincide (see figure 5(b)) and third by the following recursion: (1) take the O-point sites of BI planes from the BD (figure 5(a)) as the starting O-point set; (2) scale the current O-point set by $\tau^{2}$; (3) decorate the scaled set with BI decagon O-points; (4) repeat from step (2). A portion of the resulting quasilattice is shown in figure $5(\mathrm{~b})$. The shaded points correspond to cluster centres, which inflate with $\tau^{2}$, while the full O-point set inflates with $\tau$. One could decorate again the O-points appearing in step (3) before going back to step (2) [16] to produce the 

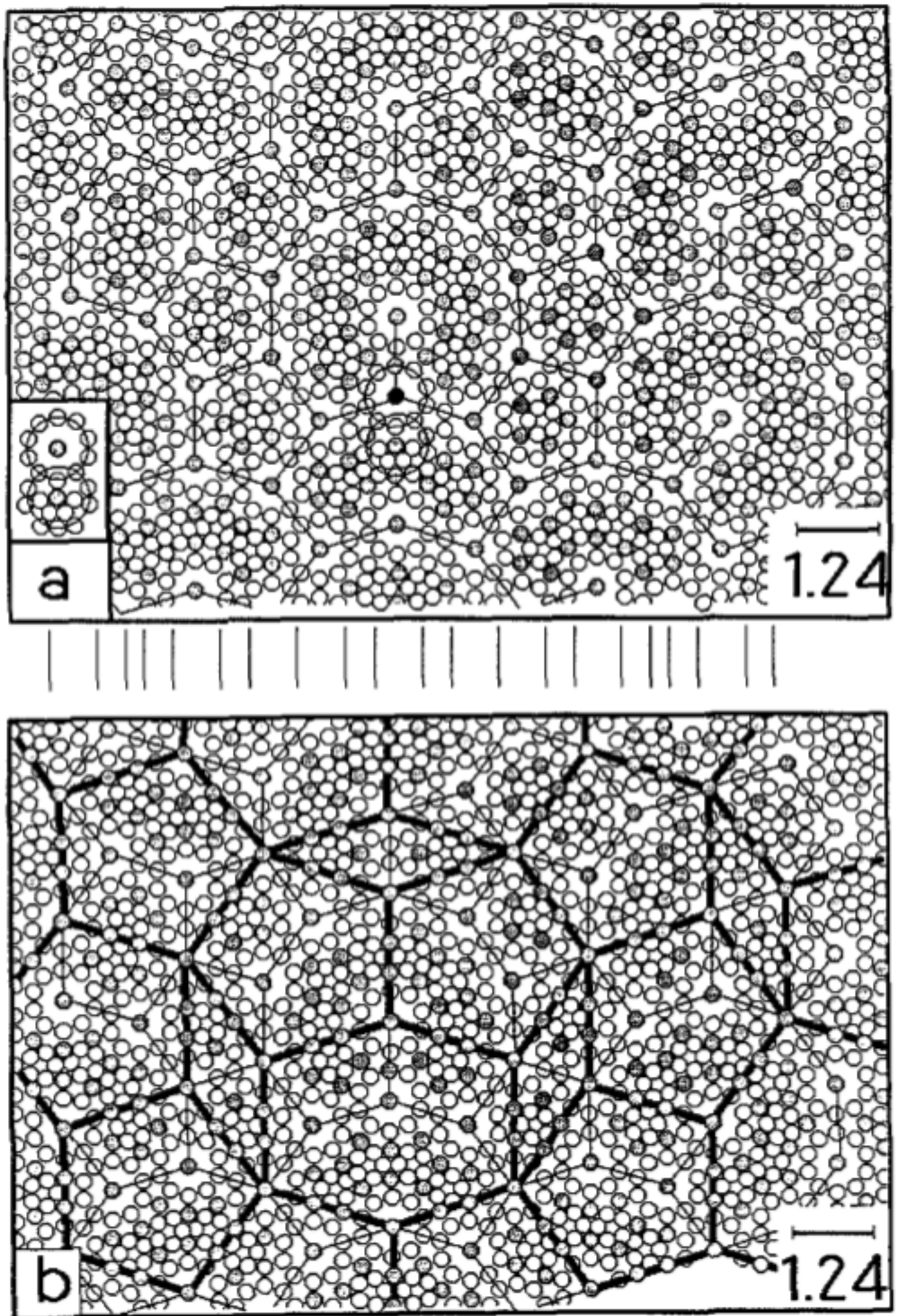

Figure 3. (a) Plane BI of an RCN with no defects. The shaded circles correspond to O-point sites, or possible cluster centres. The inset at the bottom left isolates the encircled region by the centre to show the few atomic movements required to shift a cluster centre to a nearby O-point site (phason flip). The bottom right inset shows the scale in nm. (b) QCN drawn with thicker lines over RCN in (a). Note that the tile edges are scaled by $\tau$ and that at this inflation level, the 36 thombi do not break $100 \%$ coincidence. 

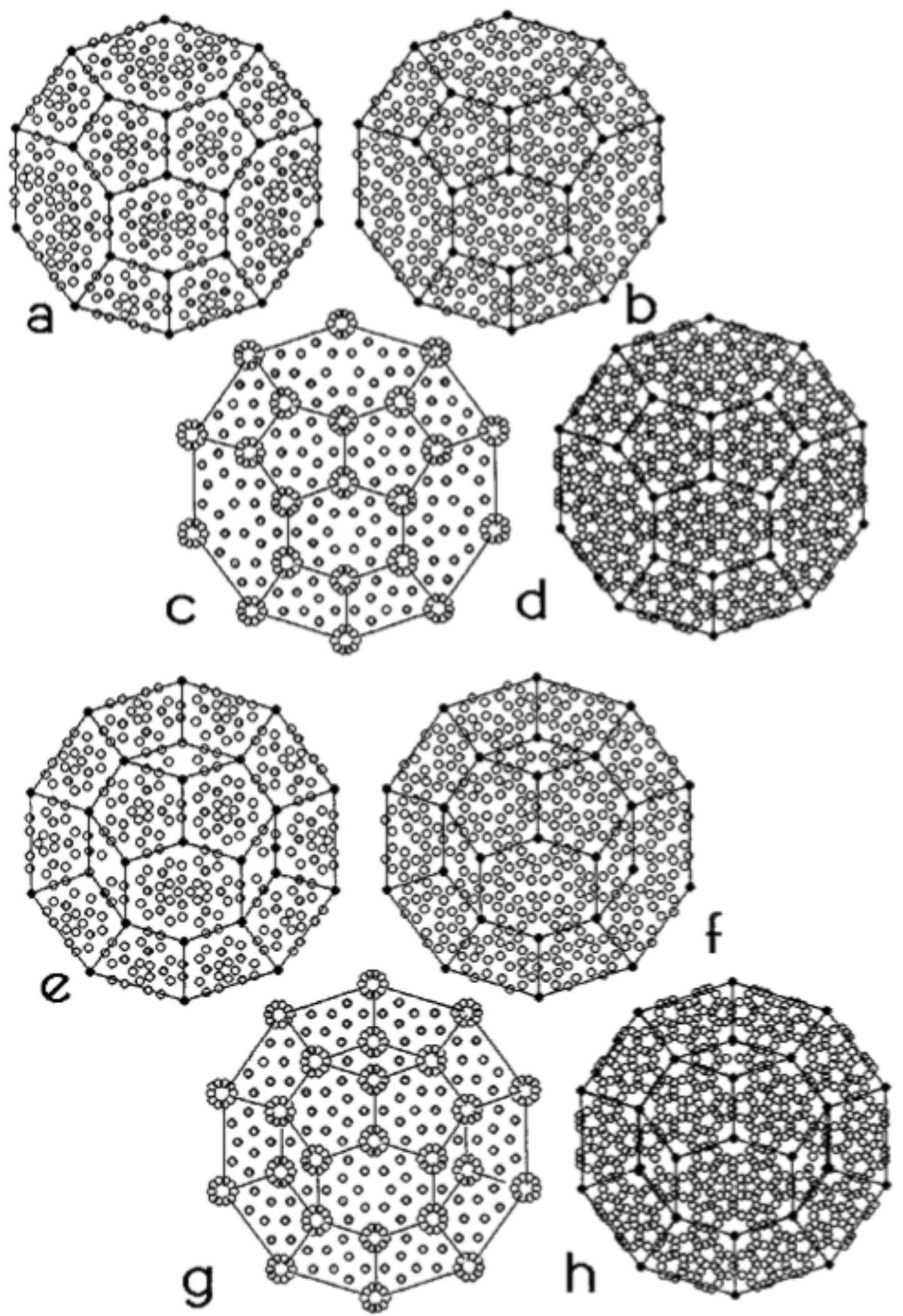

Figure 4. A complete set of planes (Bl-Al-B-A) of a $0.4 \mathrm{~nm}$ period of an unrelaxed basic decagon (see text) for two growth modes, avoiding (a) $-(d)$ and accepting (e)-(f) $36^{\circ}$ rhombi of edge length $1.2 \mathrm{~nm}$. (a), (e) BI planes; (b), (f) B planes; (c). (g) AI and A planes shown together, (d), (h) B and $\mathrm{BI}$ planes shown together to emphasize the columns of (irregular) icosahedra. Cluster centres are filled and $\mathrm{O}$-point sites shaded. Planes A and AI differ only in the orientation of the pentagons above and below cluster centres. 

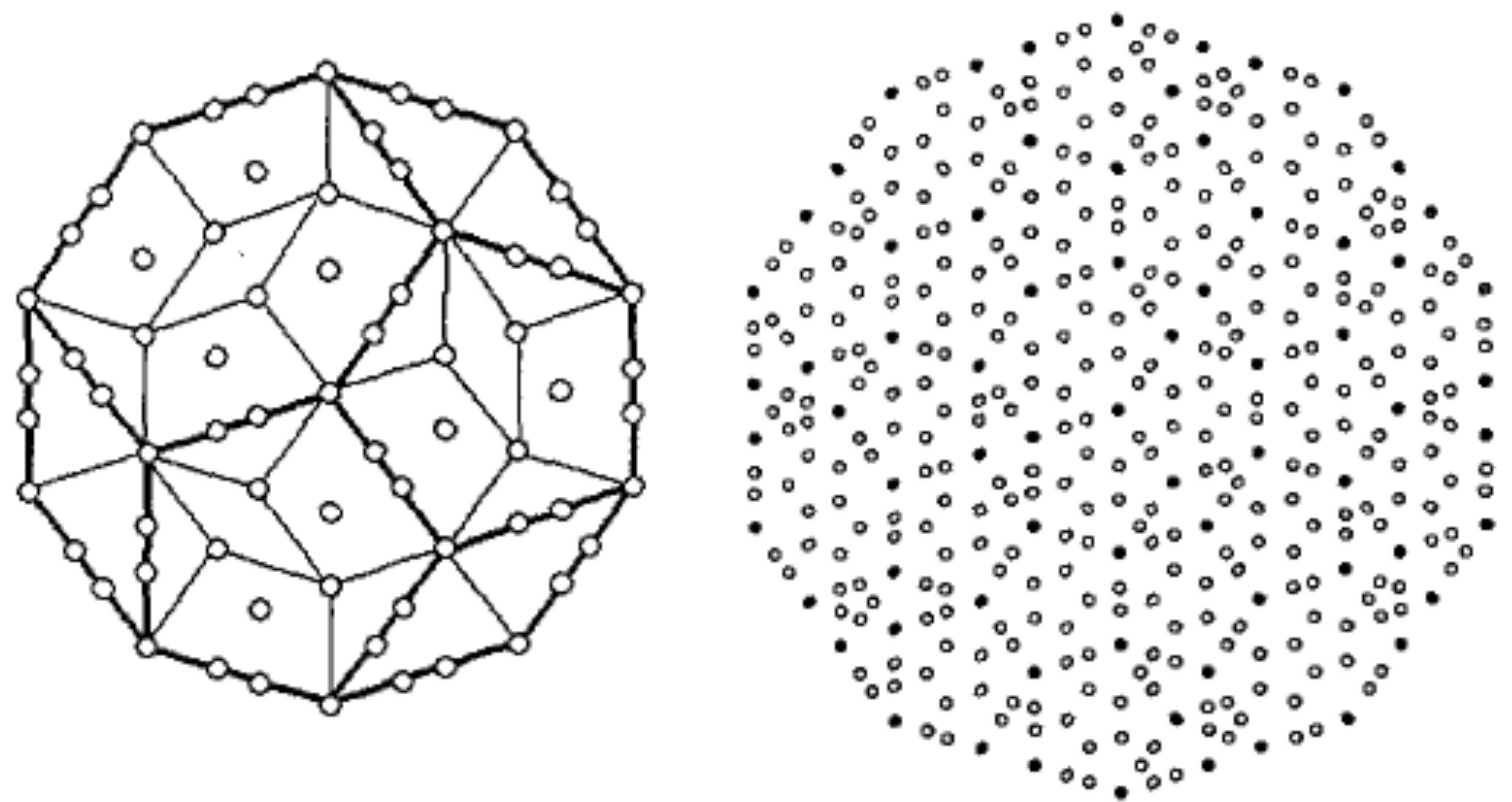

\section{a}

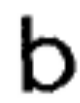

Figure 5. (a) Basic decagon with $O$-point siles. The fine lines indicate how QCN tiles (bold lines) must be decorated to obtain the quasilattice by deflation. (b) Section of a quasilattice obtained from the basic decagon and recursive nule (see text). Filled circles are cluster centres and open circles $\mathrm{O}$-point sites. The sublattice of cluster centre inflates with $\tau^{2}$ while the complete quasilattice intlates with $\mathrm{r}$.

set of all O-point sites accessible via a cluster linkage from another. Normally, this extra step cannot be repeated further since the $100 \%$ coincidence condition would be violated. The resulting quasilattice, which is the set of all possible O-point sites, is almost identical to the projection of a 3D icosahedral lattice into $2 \mathrm{D}[29]$, and can be used to generate RCNs by randomly selecting from it cluster centres meeting the $100 \%$ coincidence requirement. If microcrystalline domains are avoided, or kept very small as in RCNI, a $Q C N$ is defined. Different $Q C N S$ are equivalent, in a cut and projection scheme, to different shifts of the strip along $E^{\perp}$, an operation that does not change the local isomorphism.

\section{Final remarks}

Based on the DRM model, we have described the D phase and all observed approximants by a random accretion of clusters following a simple set of packing rules. Some words can be said about the mechanism that can produce the transformation from $D$ to the approximant phase, and the condition under which such transformations may occur.

It has been pointed out [8], that the crystal-quasicrystal transformation may occur through phasons. This point of view is supported by our results. A phason hop or flip is defined as a shift of a network node (cluster centre) into an adjacent O-point. Only a few small diffusive atomic displacements (see the inset in figure 3 and [30]) are required to produce the phason flips necessary to move a cluster centre to an adjacent O-point site, provided that the atoms move cooperatively.

Phason hops must occur between O-points to preserve the required maximum coincidence ratio. Such movements should occur randomly at high temperature, increasing 
the configurational entropy and reducing the size of crystalline domains. A reduction of the size of microcrystalline domains increases the Shannon-entropy of the system. The relevance of the Shannon-entropy in crystal-quasicrystal phase transitions has been already suggested [31-34]. This entropy can be calculated for arbitrary structures in either physical or perpendicular space and measures the homogeneity of the system, reaching a maximum in the quasicrystalline state where there are no microdomains [35]. It must be noted that the quasicrystalline state is a possible configuration in the random tiling ensemble. Numerical values for D RCNs will be given elsewhere [36]. It is therefore likely that the microcrystaltine-D transformation is produced by a randomization of the structure at high temperature trough phason flips like these. Note that around every cluster centre there is always a large number of 'unfilled' O-points to choose for the node to hop into, so disorder can easely percolate through the whole lattice.

The above flips can not only produce a rearrangement of the tiles but also change their geometry into any shape compatible with the DRMs cluster geometry and packing rules. Note that a simple rearrangement of tiles cannot explain the quasicrystal-crystal transformation observed in $\mathrm{AlCoCu}$ [7], where pentagons, hexagons and $36^{\circ}$ rhombi, give way to $72^{\circ}$ rhombi. To shed some light into the phase transformation problem, ground state calculations of RCNs based on simple Lennard-Jones potentials are currently under way. Such calculations depend on the composition of the system, and on the chemical content of each atomic site, which can in principle be deduced from symmetry and elastic energy (determined through atomic sizes) arguments. Also, diffraction calculations are being initiated using a general theory for interpenetrating clusters [37].

\section{References}

[1] Levine D and Steinhardt P J 1984 Phys, Rev, Lett, 532477

[2] Kalugin P A, Kitaev A Yu and Levitov L S 1984 JETP Lett. 64200

[3] Onoda G Y, Steinhardt P H, DiVicenzo D P and Socolar J E S 1988 Phys, Rev. Lett. 602653

[4] Henley C 1991 in Quasicrystals, the State of the Art ed D P Di Vicenzo and P J Steinhardt (Singapore: World Scientific) p 429, and references therein

[5] Audier M, Bréchet Y, de Boissieu M, Guyot P, Janot C and Dubois J M 1991 Phil. Mag. B 631375

[6] Audier M, Launois P, Dénoyer F, Lambert M, Dong C and Dubois J M 1990 Microsc. Micrnanal. Microstruct. 1417

[7] Hiraga K. Sun W and Lincoln J 1991 Japan. J. Appl. Phys. 30 L302

[8] Audier M and Robertson B 1991 Phil. Mag, Lett. 64 401

[9] Calvayrac Y, Quivy A, Bessière M, Lefebvre S, Cornier-Quinquandon M and Gratias D 1989 Phil. Mag. B 60855

[10] Romeu D 1987 Mater. Sci. Forum 22-24 257

[11] Romeu D 1988 lnt. J. Mod. Phys. B2 265

[12] Romeu D 1988 int. J.Mod. Phys. B2 77

[13] Romeu D 1990 Acta Metall. Mater. 38113

[14] Aragón J L, Romeu D and Gómez A 1991 Phys. Rev. B 44584

[15] Romeu D 1993 Phil. Mag. B 6777

[16] Romeu D 1993 J. Non-Cryst. Solids 153-154 232

[17] Dong C, Dubois J M, Kang S S and Audier M 1992 Phil. Mag. B 65107

[18] Hiraga K, Sun W, Lincoln J, Kaneko M and Matsuo Y 1991 Japan. J. Appl. Phys. 302028

[19] Burkov S E 1991 Methods of Structural Analysis of Modulated Struchures and Quasicrystals ed J M PérezMato, F J Zániga and G Madariaga (Singapore: World Scientific) p 531, and references therein

[20] Kumar V, Sahoo D, Anthithan G 1986 Phys. Rev. B 346924

[21] Yamamoto A and Hiraga K 1988 Phys. Rev. B 376207

[22] Li Z and Kuo K H 1991 Proc. China-Japan Seminars on Quasicrystals ed K H Kuo and T Ninomiya (Singapore: World Scientific) p 103

[23] Daulton T L, Kelton K F and Gibbons P C 1993 J. Non-Cryst. Sollds 153-154 15 
[24] Kortan A R, Becker R S, Thiel F A and Chen H S 1990 Phys. Rev. Left. 64200

[25] Ranganathan S 1966 Acta Crystallogr, 42197

[26] Becker R S and Kortan A R 1991 Quasicrystals, the State of the Art ed D P DiVicenzo and P I Steinhardt (Singapore: World Scientific)

[27] Romeu D and Dingley D J 1993 to be published

[28] Steurer W and Kuo K H 1990 Acta Crystallogr. B46 703

[29] Shaoking W and Hengqiang Y 1992 Phil. Mag. Lett. 6549

[30] Romeu D and Aragón J L 1993 Crystal-Quasicrystal Transitions ed M J Yacamán and M Torres (New York: Elsevier) p 193

[31] Torres M, Pastor G, Jiménez I, Aragón J L and Jose-Yacamán M 1990 Phil. Mag. Lett. 62349

[32] Torres M, Aragon J L and Pastor G 1991 Scr. Metall. Mater. 251123

[33] Aragón I L and Torres M 1991 Europhys, Lett. 15203

[34] Torres M 1991 Methods of Structurul Analysis of Madulated Structures and Quasicrystals ed J M Perez-Mato. F J Zúniga and G Madanaga (Singapore: World Scientific) p 620

[35] Torres M, Pastor G, Jiménez I, Aragón J L and Romen D 1992 Scr. Metall. Mater. 2783

[36] Torres M, Jimenez I and Romeu D 1993 to be published

[37] Verger-Gaugry J L 1991 J. Physique I 11303 